\title{
O mundo não é feito só de triângulos ou de Oceano Atlântico: A teoria pós-colonial e o Atlântico Sul "Lusófono"
}

\author{
The World is not only made os the triangles or \\ the Atlantic Ocean: Postcolonial theory and the \\ "Lusophonic" South Atlantic
}

\author{
ANTHONY SOARES
}

RESUMO: NEIL LAZARUS NOTOU QUE O APARECIMENTO DOS ESTUDOS PÓS-COLONIAIS COMO DISCIPLINA COINCIDIU COM O "FIM IMPOSTO À 'ERA BANDUNG'. O PRESENTE TRABALHO ANALISA O ATLÂNTICO SUL "LUSÓFONO" COMO UM ESPAÇO DENTRO DE UM EIXO TRICONTINENTAL MAIS AMPLO QUE LEVANTAA QUESTÃO SE ESTAMOS A TESTEMUNHAR UMA RESISTÊNCIA TIPO "BANDUNG" À HEGEMONIA DO NORTE, OU APENAS UMA VERSÃO LUSO-TROPICAL DO CAPITALISMO GLOBAL O CAPITALISMO GLOBAL COM UM ROSTO MERIDIONAL RISONHO?

ABSTRACT: NEIL LAZARUS HAS NOTED THAT THE EMERGENCE OF POSTCOLONIAL STUDIES AS A DISCIPLINE COINCIDED WITH "THE ENFORCED END OF THE 'BANDUNG ERA'". MY WORK EXAMINES THE "LUSOPHONE" SOUTH ATLANTIC AS A SPACE WITHIN A WIDER TRICONTINENTAL AXIS THAT RAISES THE QUESTION AS TO WHETHER WE ARE WITNESSING A BANDUNG - LIKE RESISTANCE TO NORTHERN HEGEMONY, OR MERELY A LUSO-TROPICAL VERSION OF GLOBAL CAPITALISM - GLOBAL CAPITALISM WITH A SMILING, SOUTHERN FACE?

PALAVRAS-CHAVE: TEORIA PÓS-COLONIAL, TRICONTINENTALISMO, ATLÂNTICO SUL LUSÓFONO KEYWORDS: POSTCOLONIAL THEORY, TRICONTINENTALISM, LUSOPHONE SOUTH ATLANTIC

* Research \& Policy Manager, Centre for Cross Border Studies. Professor de Estudos Portugueses (2001-2013) e Fundador e Diretor do Postcolonial Research Forum (2004-2013) na Queen's University Belfast (Reino Unido). 
a verdade, este estudo representa um pedido de ajuda. Ajuda ou para descobrir um caminho capaz de resolver uma tensão inerente à teoria pós-colonial, ou para confirmar que essa tensão é uma necessidade produtiva cuja resolução não faz sentido. Um vértice dessa tensão resulta de uma aparente tendência dentro da área pós-colonial de rasurar aquilo que pretende analisar, aplicando uma visão crítica baseada em binarismos reificados que torna invisível toda uma gama de micro-histórias que contrariam as simples oposições entre colonizador e colonizado. Como muitos reconhecem, também tende a subsumir dentro de um modelo específico do colonialismo e imperialismo práticas - tais como as portuguesas - que não podem ser verdadeiramente acomodadas sem serem distorcidas. O outro vértice que forma esta tensão é precisamente uma reação ao seu polo "contrário" e - através da sua problematização daquilo que é visto como um modelo simplístico e a-histórico que posiciona sujeitos em um de dois campos em eterna oposição - oferece como alternativa uma vasta gama de instâncias pontuais de experiências coloniais e pós-coloniais, atentas aos seus respetivos contextos geográficos e históricos, e à possibilidade de permeabilidade entre os significantes "colonizador" e "colonizado".

Neste caso o problema não advém necessariamente da contraposição de uma versão atomística da teoria pós-colonial fundada numa série de particularismos, visando operar contra uma tendência mais totalizadora que pensa o colonialismo como fenómeno de aplicação geral e contra o qual põe em jogo um fenómeno de anticolonialismo de natureza igualmente generalizável. Aliás, no seu ensaio "Apontamentos para conceptualizar uma Europa pós-colonial”, Paulo de Medeiros - após ter discutido "a favor de uma atenção particular às especificidades históricas dos vários colonialismos, com destaque para a situação portuguesa" - deixa claro a sua posição em relação à tentação de se concentrar exclusivamente no caso pontual: "nada se me afigura de mais pernicioso que o cair de novo numa espécie de isolacionismo avulso" (MEDEIROS, 2006, p. 348). Pelo contrário, aquilo de que necessitamos é uma análise atenta às "condições coloniais e pós-coloniais da sociedade portuguesa na sua diferença em relação com outros colonialismos e em comparação com eles" (MEDEIROS, 2006, p. 349). Contudo, é precisamente esta questão de diferença dentro da teoria pós-colonial que eu vejo como sendo a fonte de tensão, especialmente quando se crê ser necessário ir para além de 
uma conjuntura analítica que nos oferece apenas comparações entre casos pontuais, para uma abordagem capaz de tirar conclusões dos seus aspetos relacionais; ou, nas palavras de Roberto Vecchi, "uma direcção analítica que do particular e do detalhe se expanda rumo a uma generalização que talvez (...) proporcione alguns elementos não de comparação mas pelo menos de leitura combinada dos factores em jogo" (VECCHI, 2010, p. 131).

Talvez o Oceano Atlântico nos possa servir como um espaço com as dimensões suficientes para chegar a uma útil compreensão geral da diversidade histórica que ele contém. Na sua introdução a Cultures of the Lusophone Black Atlantic, os organizadores reivindicam de modo positivo um estudo dos mares como espaços de produção cultural, apesar dos riscos inerentes a um empreendimento de tal natureza:

Abundam os perigos ocultos porque, como espaços históricos e culturais, os mares não possuem limites visíveis, marcos inscritos ou donos de título legítimo; no entanto, tais perigos têm a possibilidade - e oferecem na realidade - novas oportunidades. Os mares srgem como unidades inteiras e contínuas que podem absorver e refletir a história humana com a mesma plasticidade que absorvem e refletem a luz do sol. Permitem-nos suspender momentaneamente as nossas dicotomias rígidas e dogmáticas entre nações, civilizações, Nós e Eles, Europa e África, o Ocidente e o Resto (NARO, SANSI-ROCA E TREECE, 2007, p.1; esta e todas as outras traduções são da minha responsabilidade).

Os mares, com a sua falta de limites, sua plasticidade, tornam-se espaços nos quais é-nos possível abandonar a tendência de reificar especifidades autossuficientes, para nos tornarmos atentos a como os oceanos têm testemunhado o desenvolvimento recíproco de uma modernidade que - no caso que aqui nos interessa - Nancy Priscilla Naro e seus colegas propõem denominar uma modernidade Atlântica ('Atlantic modernity') (NARO, SANSI-ROCCA E TREECE, 2007, p. 5). Neste sentido não se trata de uma modernidade construída pelo "Ocidente", imposta "no resto", e resistida por um Atlântico Negro ou do Proletariado, mas sim uma em que todas estas identidades "podem de facto ser essenciais à (...) modernidade" e "constituem uma parte integral do mundo moderno" (NARO, SANSI-ROCCA E TREECE, 2007, p. 5). Mas o que será este "mundo moderno" a partir do qual estamos a falar, e que tantas micro-histórias ajudaram a construir de modos tão diversos e nem sempre necessariamente conflituais ou oposicionais? Para descobrir se 
nos poderá ajudar a responder a esta pergunta - e se fará qualquer sentido em uma análise pós-colonial - iremos agora examinar um local específico no Oceano Atlântico que liga dois espaços "lusófonos" em cada um dos seus continentes do Sul.

O Bloco 15/06 é um local de exploração petrolífera situado ao largo da costa do norte de Angola, em que interesses brasileiros e angolanos têm representação através - respetivamente - da Petrobras e Sonangol. Contudo, logo à partida temos de estar atentos a uma tentação interpretativa que nos leve a generalizar com base nos casos pontuais da Petrobras e da Sonangol para daí chegar ao Brasil e a Angola, e assim a conclusões abrangendo ambas nações, conclusões essas que se baseiam em duas empresas comerciais. Uma companhia estatal formada em 1976 após a nacionalização da Angol, a Sonangol é a "concessionária exclusiva para a exploração de hidrocarbonetos líquidos e gasosos no subsolo e na plataforma continental de Angola", evidenciando na declaração da sua missão uma preocupação em se apresentar como simultaneamente competitiva no mercado global e trabalhando a favor dos interesses da sociedade angolana: a empresa tem "por finalidade promover o desenvolvimento harmonioso do país e reforçando a utilização sustentável dos recursos nacionais e hidrocarbonetos" (SONANGOL, 201X). De modo semelhante, 64\% da Petrobras - criada em 1953 durante o regime de Getúlio Vargas (e no mesmo ano em que a Angol foi fundada como uma subsidiária da companhia portuguesa, Sacor) - está nas mãos do Estado Brasileiro e, embora apenas minimamente de início, tem mantido uma presença em águas angolanas desde 1979. Tal ligação comercial entre Brasil e Angola poderá parecer fazer eco a uma relação direta entre os dois territórios que já era evidente durante o tráfico transatlântico de escravos, uma atividade que, de acordo com Roquinaldo Ferreira, "ocorreu dentro de uma conjuntura bilateral que minava a influência portuguesa enquanto fortalecia as ligações diretas de Angola com o Brasil" (FERREIRA, 2007, p. 100). Aliás - e sem querer necessariamente confundir aventuras comerciais com atividades de outra natureza - o envolvimento da Petrobras no Bloco 15/06 (entre outros) poderia ser contemplado no contexto mais alargado de uma presença brasileira no "Sul global", como atesta uma manchete no Correio do Brasil, que alertava os seus leitores para o facto que o "Brasil trabalha para acelerar a cooperação Sul-Sul”. O artigo explicava que em 2011 a "Agência de Cooperação Brasileira 
[estava] participando (...) de uma série de projetos econômicos (...) em mais de 80 países em desenvolvimento na África, na Ásia, na América Latina e no Caribe" (CARTA MAIOR, 2011). Enquanto a Petrobras explora as águas do Atlântico ao largo da costa angolana, o governo brasileiro - através da Agência de Cooperação Brasileira - tem participado em uma série de projetos de "desenvolvimento" em Angola, abrangendo áreas como a educação, saúde, agricultura e cultura (AGÊNCIA DE COOPERAÇÃO BRASILEIRA, 2011, p. 15-24). Esta relação do Sul lusófono é, nas palavras do então Ministro das Relações Exteriores brasileiro, parte de uma presença estratégica do Brasil na África que representa "ao mesmo tempo parte do esforço de concretização da vocação universalista do País e de resgate da identidade nacional na formulação de política externa" (AMORIM, 2011, p. 5).

Deixando à parte considerações quanto à possível relevância de um discurso invocando uma vocação universalista e a necessidade de resgatar a identidade nacional brasileira, voltemos ao Bloco 15/06. Enquanto o envolvimento da Petrobras e da Sonangol poderá ter a aparência de uma relação que atravessa um Atlântico Sul "Lusófono" no qual não há necessidade de um triângulo cujo principal eixo ficaria situado na Europa, não é esse necessariamente o caso. Embora Portugal não esteja presente no Bloco 15/06, uma empresa Italiana - a ENI Angola - tem a maior participação, com outras companhias participantes sendo a Total da França e a Statoil norueguesa. Poderíamos até considerar a existência de um triângulo construído com base numa estrutura de poder de sentido inverso àquele tido como "tradicional", pois a Sonangol é uma das empresas com maior participação na Galp Energia de Portugal e a Galp - por sua vez - retinha uma participação de 9\% no Bloco 14. No entanto, a ideia de um triângulo terá de ser imediatamente descartada para que possamos incluir outra área do Atlântico - também do Norte - representada pela Falcon Oil, dos Estados Unidos, que retém 5\% do Bloco 15/06.

Mas a existência de outra companhia - a Sonangol Sinopec International (SSI) - com a segunda maior participação no Bloco 15/06 (de 20\%), obriga-nos a olhar para além do Atlântico. A SSI foi criada em 2004 como uma "joint venture" pela Sinopec, com esta sendo uma das principais companhias petrolíferas na China e retendo $55 \%$ da SSI. Os outros $45 \%$ pertenceriam aparentemente à Sonangol, fazendo com que este fosse um negócio conjunto entre a Sinopec e a empresa estatal angolana. Contudo, em 2010, após uma re- 
organização interna da Sinopec que resultou na divulgação na bolsa de várias informações sobre a estrutura da empresa, foi revelado que os outros $45 \%$ da SSI pertenciam à "China Sonangol". Nunca foi divulgada a propriedade da China Sonangol, mas - apesar do nome - crê-se que não pertence nem ao estado chinês nem à Sonangol. Ela parece ser uma criação da orientação política da China em 2002 de "sair" ("going out") que incentiva empresários chineses do setor privado a embarcarem em atividades no estrangeiro, e a China Sonangol poderia ser descrita como um consórcio de "empresários" chineses, africanos e de outros paradeiros. No entanto, mesmo que a propriedade da China Sonangol seja tema discutível, o seu discurso público invoca um desejo de minar a hegemonia do "Norte", referindo-se à "cooperação Sul-Sul" como uma das suas missões e à introdução "na China de conceitos louváveis e ideias meritórias de aprendizagem provenientes de outros países em desenvolvimento" (CHINA SONANGOL, 2013).

Referências à cooperação Sul-Sul originando em duas das nações representadas no Bloco 15/06 no Atlântico poderão aparentar formar parte das características que Robert Young aponta para o Tricontinentalismo, ou seja, o seu "rico património, o legado radical da sua determinação política, a sua rejeição do status quo" (YOUNG, 2001, p.428). De facto, Sam Pa, o homem considerado o criador da China Sonangol, parece encarnar as relações entre a América Latina, África e Ásia que seriam essenciais ao Tricontinentalismo. Em 2004, por exemplo, após ter visitado o presidente argentino, Néstor Kirchner, o Senhor $\mathrm{Pa}$ apareceu no programa de televisão Aló Presidente, de Hugo Chávez, enquanto que as suas relações com o presidente angolano, José Eduardo dos Santos, remontam aos tempos em que ambos estudaram na ex-União Soviética, relações essas que foram fortalecidas graças a negócios empreendidos entre o Sr. Pa e o MPLA durante a guerra civil angolana. Já a própria China Sonangol opera a partir do princípio que irá construir infraestruturas em Angola utilizando as receitas que recebe do comércio de petróleo angolano - seguindo assim a filosofia chinesa de "todos a ganhar" ("win-win"). Contudo - e como talvez já era de suspeitar pelo menos no caso da China Sonangol se não mesmo em relação ao envolvimento brasileiro no petróleo angolano - a realidade é que esta versão do Tricontinentalismo é apenas uma deformação do seu espírito original. Enquanto estimativas dos lucros da China Sonangol como resultado das suas operações em Angola 
chegam a largos milhares de milhões de dólares, os seus gastos em infraestrutura angolana não vão além de algumas centenas de milhões, com a baixa qualidade dos seus projetos ficando muito evidente após a abertura do Hospital Geral de Luanda, que encerrou pouco depois para possibilitar obras de emergência.

Assim, o que é que o Bloco 15/06 nos diz afinal sobre o nosso mundo "moderno", e será ele apropriado para uma análise pós-colonial? Em primeiro lugar, ao simplesmente identificar as empresas que terão investido nele, podemos observar que elas não nos levam apenas a vários pontos limítrofes do Oceano Atlântico, mas também para além dele. No entanto, este campo multidirecional não leva em conta outras particularidades que mereciam ser analisadas, tal como as origens da mão de obra ou as correntes de conhecimento tecnológico sendo empregues no Bloco 15/06. Em segundo, mesmo se o Brasil e a Angola - nações outrora colonizadas por Portugal - sejam de maior importância ao Bloco 15/06, poderá uma perspetiva pós-colonial lusófona fazer mais do que simplesmente traçar trajetórias históricas que levaram a um presente abrangendo um mundo de dimensões mais largas - um mundo onde o lusófono (ou o francófono, hispanófono ou mesmo anglófono) perde face ao triunfo de um sistema global impelido por imperativos que talvez tornem essas distinções irrelevantes?

Como resposta porventura algo provocadora a essa pergunta, gostaria de passar a uma breve leitura do artigo de Neil Lazarus, "What Postcolonial Theory Doesn't Say” (“O que a Teoria Pós-colonial não diz”). Nesse trabalho o autor começa por delinear uma corrente dos estudos pós-coloniais representativa de "uma racionalização e ajustamento pragmático ao desaparecimento das ideologias que teriam florescido durante os anos 'Bandung”, e que após a queda do Muro de Berlim "estava disposta a pronunciar a morte definitiva do Marxismo" (LAZARUS, 2011, p. 5). Se esta fosse a única linha de pensamento dentro dos estudos pós-coloniais que - atenta às condições presentes - julga (talvez com toda a razão) os movimentos anticoloniais, anti-imperiais e tricontinentais terem sido falhanços inevitáveis, então uma perspetiva pós-colonial lusófona só teria utilidade como ferramenta para uma análise histórica. Quer isto dizer que ela poder-nos-á fornecer uma visão de como as práticas coloniais portuguesas diferiam de outras, e como a resistência a essas práticas operaram nas suas variadas formas, mas limitados pelo 
facto que quaisquer que tiverem sido essas diferenças, elas nos touxeram ao mesmo presente para além do qual já não têm verdadeira importância.

Contudo, no seu artigo Lazarus aponta para uma corrente alternativa dentro do campo pós-colonial - uma alternativa que representa "uma iniciativa assumidamente progressiva ou radical, que se opõe às formas dominantes assumidas pela orientação política e discurso antilibertários nos anos negros desde meados da década de 1970" (LAZARUS, 2011, p. 5). É esta corrente que mais adequadamente encarna a "rejeição do status quo" que - como já mencionei - Robert Young vê como o legado que nos deixou o Tricontinentalismo. No entanto Neil Lazarus atesta que até esta forma de crítica pós-colonial "tem sido repetidamente comprometida por um erro categórico ao centro da sua prática crítica":

O problema é simplesmente que os estudiosos da disciplina tendem a não prestar a devida atenção ao facto que o colonialismo faz parte integral de uma dinâmica histórica englobante de maiores dimensões, que é o capitalismo na sua trajetória global. Tem havido uma notável falência na disciplina em situar os projectos históricos do colonialismo e imperialismo dos contextos determinantes da gênese, consolidação e do desenvolvimento do sistema mundial moderno (LAZARUS, 2011, p. 7).

Tal falência, quanto a Lazarus, leva a interpretações do imperialismo em que - e utilizando Said como exemplo - é "tipicamente representado como conjuntura política e relacionado, em termos civilizacionais, ao 'Ocidente' em vez do capitalismo” (LAZARUS, 2011, p. 10). O que geralmente fica omitido ou encoberto em tais interpretações são as mudanças efetuadas aos meios de produção e aos regimes de acumulação que resultaram no modelo económico que hoje predomina. Assim, para Lazarus:

É (...) importante insistir (...) que de tudo aquilo que poderia ter envolvido - ou mesmo aquilo que realmente envolven - desde a sua aniquilação sistemática de comunidades inteiras até ao cultivo de gostos e preferências estéticas - o colonialismo como processo histórico envolveu a integração forçada no sistema mundial capitalista de sociedades até então não-capitalizadas, ou sociedades em que o meio de produção capitalista não era hegemónico (LAZARUS, 2011, p. 11).

Mas, ao insistir nisto, não estaremos a voltar para onde começamos? Todos 
os caminhos, por mais acidentados ou diretos, levam-nos para um sistema mundial capitalista que engloba tudo, e a partir do qual, para se chegar ao Lusófono, é preciso voltar atrás. Ou seja, para poder observar a "diferença" lusófona, necessitamos voltar a um passado quando o modelo económico que parece hoje dominar o globo ainda não retinha essa posição incontestada. Por agora, o Bloco 15/06 - apesar da presença de um "Sul Lusófono" - não representa mais do que uma manifestação em águas angolanas de um sistema capitalista de tendência hegemónica (se não hegemónico de facto) em que existe uma participação brasileira. Não será aqui que iremos encontrar o Tricontinentalismo de Robert Young - se ele alguma vez verdadeiramente existiu...

Por coincidência (ou não), é o Atlântico que serve como espaço referencial quando Young no seu artigo "Postcolonial Remains" ("Restos Pós-Coloniais"), assegura-nos que a teoria pós-colonial sobrevive nos nossos tempos precisamente porque a sua morte ou inoperabilidade terá sido anunciada nos Estados Unidos e na França. "O desejo de pronunciar dos dois lados do Atlântico a teoria pós-colonial como morta sugere", de acordo com Young, "que a sua presença continua a perturbar e a provocar ansiedade: o verdadeiro problema é que o pós-colonial ainda resta" (YOUNG, 2012, p. 19). $\mathrm{O}$ autor afirma de modo categórico que o "Pós-colonialismo” não é apenas uma disciplina, ou uma teoria que chegou ou não ao seu fim", mas sim um "projeto político abrangente" que visa "reconstruir as formações de conhecimento ocidentais, reorientar normas éticas, inverter as estruturas de poder do mundo, recriar o mundo de baixo para cima" (YOUNG, 2012, p. 20). Portanto, longe de nunca ter verdadeiramente existido ou de ter morrido, o pós-colonialismo continua a reter uma capacidade de resistência cuja vitalidade significa que "o século XXI é já o século de potencialização pós-colonial" (YOUNG, 2012, p. 20). Esta situação advém em parte do facto de que os "booms económicos transformaram nações como o Brasil, a China e a Índia em novas dinâmicas que contribuem para uma deslocação dos paradigmas de poder económico e político que modificaram claramente a sensibilidade de dependência colonial" (YOUNG, 2012, p. 20). Assim, é o Atlântico Norte (representado aqui pelos Estados Unidos e França) que tenta negar um impulso regenerador e de contestação cujo vigor vem de um "Sul" que vai muito para além desse Oceano.

No entanto, podemos notar uma diferenciação importante feita entre um 
pós-colonialismo de enorme vitalidade e um Tricontinentalismo que Young descreve como um "novo tricontinental subalterno" muito aquém da "militância da Organização de Solidariedade dos Povos de África, Ásia e América Latina (OSPAAAL) desenvolvida na década de 1960" (Young, 2012, p. 26). Enquanto esta nova e triste versão do Tricontinentalismo é constituída de "emigrantes dos países mais pobres da África, Ásia e América Latina" - "uma diáspora tricontinental invisível” (YOUNG, 2012, p. 26) - a energia do pós-colonialismo tem invadido os corredores académicos com uma força que resulta na sua influência sobre "tantas disciplinas diferentes", aparentemente chegando mesmo a surpreender este pensador como confessa numa entrevista recente (SANTOS, 2013, p. 294). Contudo, é na sua identificação da composição deste "novo tricontinental subalterno" em "Postcolonial Remains", e do seu comentário quase que em forma de um aparte na entrevista que acabei de referir, no qual diz talvez ser a Economia a única disciplina praticamente imune à influência pós-colonial (SANTOS, 2013, p. 294), que nos deparamos com um problema interpretativo (para não falar do apelo de Young para uma reavaliação do imperialismo em termos da sua capacidade para oferecer a possibilidade de convivência entre diferentes identidades, exemplificado hoje em dia pelo Qatar...).

Como Benita Parry sugere na sua análise de 'Postcolonial Remains', Robert Young parece alinhar-se com aqueles que "defendem uma política de amizade no lugar de uma política de conflito e luta, substituindo assim a tradição de esquerda de solidariedade entre os oprimidos pela noção liberal de coexistência pacífica entre todos" (PARRY, 2012, p. 342). Assim, quando Young conclui que "agora a questão pós-colonial é como tornar acessível o sonho de emancipação de todos aqueles que foram excluídos das necessidades da modernidade contemporânea" (YOUNG, 2012, p. 27), não nos oferece uma solução que passe por uma oposição direta e ativa contra o sistema mundial capitalista; nunca reconhece a situação como sendo o resultado do "impacto da intrusão capitalista nas formas e instituições de sociedades pré-coloniais", ou da "continuação do domínio do capitalismo metropolitano, das estruturas de classe social e dos conflitos nos estados-nações pós-independência, e do papel do neolibralismo e de compradores nativos nos recuos de regimes pós-coloniais" (PARRY, 2012, p. 344). Na ausência de tal reconhecimento, torna-se então possível chegar a uma interpretação do Bloco 15/06 que vê a presença 
brasileira, angolana e chinesa como prova do "século de potencialização pós-colonial", invertendo o mapa de poder político e económico. O sistema, no entanto, segue o seu percurso sem sérios percalços, e até poderá apresentar uma via "Lusófona" no Sul, mas os objetivos do projeto pós-colonial como ele foi pensado na sua infância e das lutas anticoloniais que o inspiraram ficarão pelo caminho. Chegamos então à questão essencial: será que sinceramente queremos pôr fim ao sistema mundial capitalista como ele existe hoje - com tudo que isso poderá implicar - ou será que teremos que admitir que esse sistema - apesar de todos seus efeitos menos positivos - produziu um mundo que não desejamos ceder? Se concluirmos que este sistema tem de ser resistido, então existe a possibilidade de que essa resistência poderá partir (pelo menos em parte) de um "Sul Lusófono" pós-colonial - se assim o quisermos e o tornarmos realidade. Mas se admitirmos que a presente conjuntura socioeconómica global é algo do qual não queremos desistir, então a "diferença” Lusófona só se encontrará no passado. De qualquer modo precisa-se coragem e de uma triangulação mais ampla.

\section{Referências Bibliográficas}

AGÊNCIA BRASILEIRA DE COOPERAÇÃO. A cooperação técnica do Brasil para a África. Brasilia: Ministério das Relações Exteriores, 2011.

AMORIM, Celso. Prefácio. In AGÊNCIA BRASILEIRA DE COOPERAÇÃO. A cooperação técnica do Brasil para a África. Brasília: Ministério das Relações Exteriores, 2011, p. 5.

CARTA MAIOR. Brasil trabalha para acelerar a cooperação Sul-Sul. Correio do Brasil, 22 ago. 2011. Disponível em <http://correiodobrasil.com.br/noticias/politica /brasil-trabalha-para-acelerar-a-cooperacao-sul-sul/286728/>. Acesso em dez. de 2011.

CHINA SONANGOL. Our company. Disponível em <www.chinasonangol.com>. Acesso em jan. de 2013.

FERREIRA, Roquinaldo. Atlantic Microbistories: Mobility, Personal Ties, and Slaving in the Black Atlantic World (Angola and Brazil). In NARO, N.P. et al (orgs). Cultures of the Lusophone Black Atlantic. Basingstoke e Nova York: Palgrave Macmillan, 2007, p. 99-128. 
LAZARUS, Neil. What Postcolonial Theory Doesn't Say. Race \& Class, v. 53, n. 1, p. 3-27, 2011.

MEDEIROS, Paulo de. Apontamentos para conceptualizar uma Europa pós-colonial. In SANCHES, M. (org.). Portugal não é um país pequeno. Cantar o "império" na pós-colonialidade. Lisboa: Livros Cotovia, 2006, p. 339-356.

NARO, Nancy Priscilla, SANSI-ROCA, Roger, TREECE, David H. Introduction: The Atlantic, between Scylla and Charybdis. In NARO, N.P. et al (orgs). Cultures of the Lusophone Black Atlantic. Basingstoke e Nova York: Palgrave Macmillan, 2007, p. 1-15. PARRY, Benita. What is Left in Postcolonial Studies? New Literary History, v. 43, n. 2, p. 341 358, Primavera 2012.

SANTOS, Emanuelle. Postcolonial Theory Meets the Portuguese-Speaking World: An Interview with Robert J.C. Young. Ellipsis, v. 11, p. 293-315, 2013.

SONANGOL. A Sonangol EP. Disponível em: < www.sonangol.co.ao >. Acesso em jan. de 2013.

VECCHI, Roberto. O pós-colonialismo português: excepção vs. excepcionalidade? In BRUGIONI, E. et al (orgs.). Áfricas contemporâneas/Contemporary Africas. Vila Nova de Famalicão: Edições Húmus, 2010, p. 131-139.

YOUNG, Robert. Postcolonialism: An Historical Introduction. Oxford: Blackwell Publishing, 2001.

YOUNG, Robert. Postcolonial Remains. New Literary History, v. 43, n. 1, p. 19-42, Inverno 2012. 